\title{
Receptor-mediated signaling in Aspergillus fumigatus
}

\section{M. Grice*, M. Bertuzzi and E. M. Bignell}

South Kensington Campus, Imperial College London, London, UK

\section{Edited by:}

Alex Andrianopoulus, University of Melbourne, Australia

Reviewed by:

David Andes, University of

Wisconsin, USA

Nancy Keller, University of

Wisconsin, USA

${ }^{*}$ Correspondence:

C. M. Grice, South Kensington Campus, Imperial College London, Exhibition Road, London SW7 2AZ UK

e-mail: c.grice11@imperial.ac.uk
Aspergillus fumigatus is the most pathogenic species among the Aspergilli, and the major fungal agent of human pulmonary infection. To prosper in diverse ecological niches, Aspergilli have evolved numerous mechanisms for adaptive gene regulation, some of which are also crucial for mammalian infection. Among the molecules which govern such responses, integral membrane receptors are thought to be the most amenable to therapeutic modulation. This is due to the localization of these molecular sensors at the periphery of the fungal cell, and to the prevalence of small molecules and licensed drugs which target receptor-mediated signaling in higher eukaryotic cells. In this review we highlight the progress made in characterizing receptor-mediated environmental adaptation in A. fumigatus and its relevance for pathogenicity in mammals. By presenting a first genomic survey of integral membrane proteins in this organism, we highlight an abundance of putative seven transmembrane domain (7TMD) receptors, the majority of which remain uncharacterized. Given the dependency of $A$. fumigatus upon stress adaptation for colonization and infection of mammalian hosts, and the merits of targeting receptor-mediated signaling as an antifungal strategy, a closer scrutiny of sensory perception and signal transduction in this organism is warranted.

Keywords: Aspergillus fumigatus, stress, virulence, signaling

\section{INTRODUCTION}

The genus Aspergillus is comprised of environmental filamentous mold fungi which utilize decaying organic matter for metabolic energy and nutrition. Aspergillus fumigatus is the most pathogenic, and is commonly isolated as an agent of human pulmonary infections (Dagenais and Keller, 2009). In healthy individuals, mucociliary clearance and pulmonary immune defences clear the hundreds of conidia inhaled daily (Balloy and Chignard, 2009). However, medical advances in transplantation and anticancer therapies have expanded the immunosuppressed patient population, and the number of individuals infected by opportunistic organisms, such as A. fumigatus, has drastically increased (McNeil et al., 2001; Chamilos et al., 2006). For opportunistic fungal pathogens, the phenomenon of "ready-made" virulence has been postulated, whereby traits which evolved for survival in ecological niches also govern survival in susceptible immunocompromised hosts (Casadevall et al., 2003; Rhodes, 2006).

Beyond residual host immune responses, there are additional obstacles to successful colonization of the mammalian lung, including tolerance of host-facilitated stresses, such as iron starvation (Schrettl et al., 2004, 2007) and alkaline pH (Peñalva and Arst, 2004; Bignell et al., 2005; Peñalva et al., 2008). The requirement for infecting fungi to detect and respond to such extracellular cues is often essential for infectious growth, and in A. fumigatus the fungal receptors through which the extracellular environment is sensed remain largely unknown. This review discusses current knowledge on receptor-mediated signaling in A. fumigatus (Figure 1) and catalogues all of the putative seven transmembrane domain (7TMD) sensors encoded by the A. fumigatus genome (Table A1). Our analysis exposes the vast numbers of uncharacterized A. fumigatus receptor-like proteins.

\section{G PROTEIN COUPLED RECEPTORS (GPCRs) IN A. fumigatus}

In silico analyses of fungal genome sequences have identified genes encoding putative GPCR proteins. In the phytopathogenic fungus Magnaporthe grisea, a screen of the predicted proteome using all GPCR sequences at the time available in the GPCR Database (GPCRDB) (Horn et al., 2003) yielded 14 GPCR-like sequences (Kulkarni et al., 2005). A similar exercise applied to A. fumigatus identified 15 putative GPCRs (Lafon et al., 2006).

In Aspergilli, putative GPCRs are classified by homology, and according to a convention established by Lafon et al. (2006) in A. nidulans, into nine groupings. In A. fumigatus, Classes 1 and 2 are comprised, respectively, of two putative pheromone receptors GprA (AFUA_3G14330) and GprB (AFUA_5G07880); Class 3 is comprised of two putative carbon sensors GprC (AFUA_7G04800), GprD (AFUA_2G12640); Class 4 is comprised of three putative nitrogen sensors GprF (AFUA_5G04100), GprG (AFUA_1G11900), and GprJ (AFUA_1G06840); Class 5 of three putative cAMP receptors GprH (AFUA_5G04140), GprI (AFUA_3G00780), and GprL (AFUA_3G01750), the latter being unique to A. fumigatus; Class 6 is comprised of a single putative GPCR, GprK (AFUA_4G01350) having a regulator of G-protein signaling (RGS) domain, unique to filamentous fungi; Class 7 includes two putative GPCRs with homology to rat growth hormone-releasing factor receptors (Miller et al., 1999) only one of which is found in A. fumigatus, GprM (AFUA_7G05300); Class 8 is comprised of three putative GPCRs with identity to yeast Izh zinc regulators 


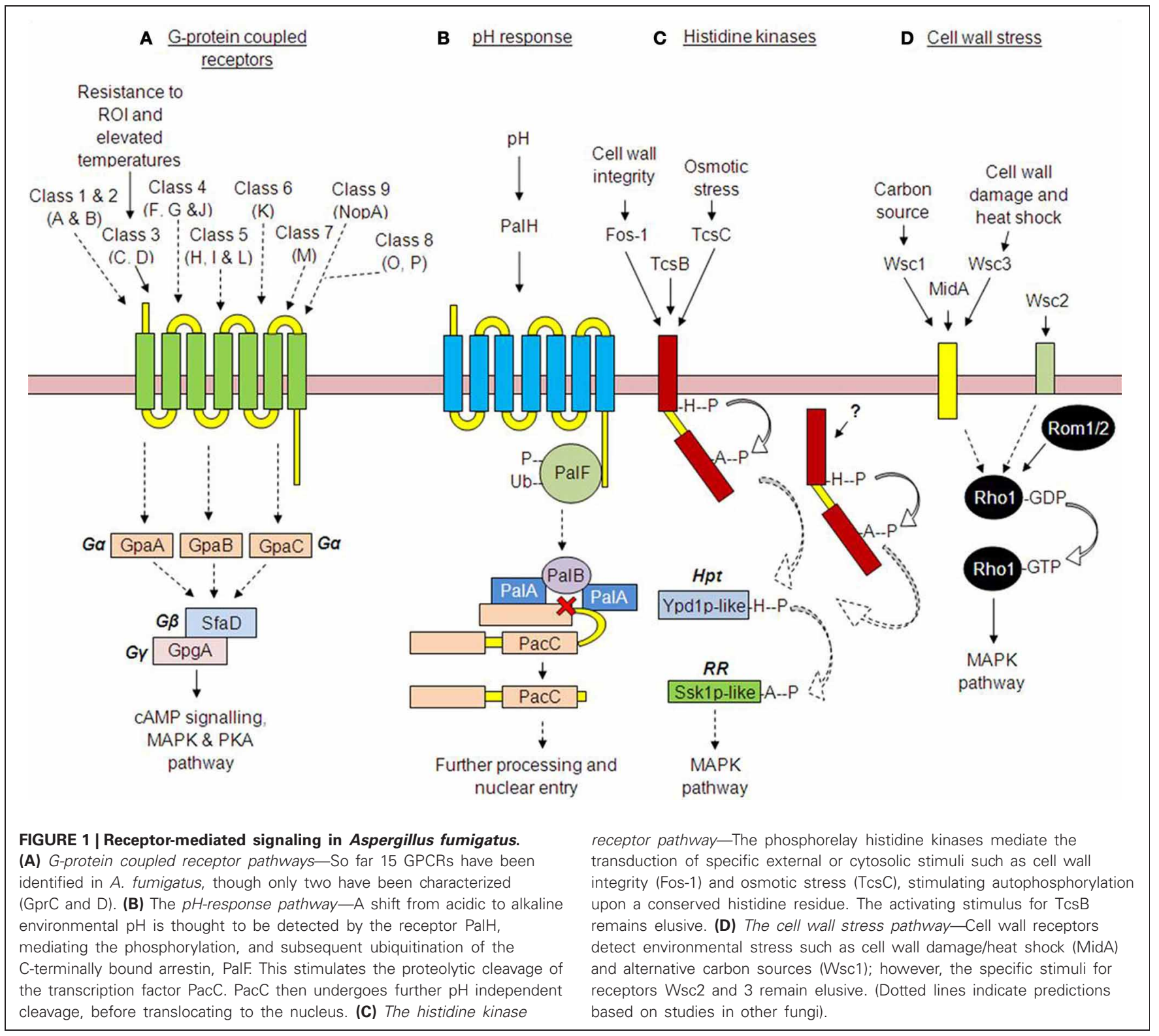

(Karpichev et al., 2002; Lyons et al., 2004), two of which are found in A. fumigatus GprO (AFUA_3G10570) and GprP (AFUA_6G07160), and Class 9 is comprised of a single putative GPCR, NopA (AFUA_7g01430) having identity to bacterial opsins. The roles of some of these receptors have been identified in other species though in A. fumigatus little is known (Figure 1).

Among the 15 predicted GPCR-like proteins in A. fumigatus, only two, GprC (AFUA_7G04800) and GprD (AFUA_2G12640), have been characterized (Gehrke et al., 2010). GprC and GprD have been noted as having homology to Gprlp of Saccharomyces cerevisiae which activates the cAMP pathway in response to glucose, as demonstrated by cAMP enzyme immunoassay (Yun et al., 1998; Kraakman et al., 1999). Furthermore, the A. nidulans GprD homologue mediates increase of intracellular cAMP in response to oxygenated polyunsaturated fatty acids (oxylipins), which act as autocrine and paracrine mediators in eukaryotic organisms (Affeldt et al., 2012). Deletion of A. fumigatus GprC and GprD resulted in significant growth impairment under all tested growth conditions and analysis of virulence revealed significant attenuation of virulence for $\triangle g p r D$ and delayed mortality for $\Delta g p r C$ in a murine model of aspergillosis (Gehrke et al., 2010). The remainder of the putative A. fumigatus GPCRs remain to be investigated and nothing is known about their molecular linkages to multi-subunit G-proteins. Unlike most Aspergillus spp. where four predicted $\mathrm{G} \alpha$ subunits occur, only three (GpaA, AFUA_1G13140, GpaB, AFUA_1G12930, and GpaC, AFUA_3G12400) have been identified for A. fumigatus (Liebmann et al., 2003), which presumably act via interaction with the G $\beta$ and $G \gamma$ subunits (SfaD, AFUA_5G12210 and GpgA, AFUA_1G05210). In the current absence of other identified $G$ protein subunits, or similar proteins, it is thought that 
the aforementioned five proteins service the entire $A$. fumigatus GPCR repertoire (Figure 1). Undoubtedly the relevance of A. fumigatus $\mathrm{G} \beta$ and $\mathrm{G} \gamma$ subunits for viability and vegetative growth is significant as $\Delta s f a D$ and $\triangle g p g A$ gene deletion mutants are extremely impaired for germination and vegetative growth (Shin et al., 2009).

\section{GENOME-WIDE in silico PREDICTIONS OF A. fumigatus INTEGRAL MEMBRANE PROTEINS}

Kulkarni et al. (2005) noted, based upon membrane topology, that the number of putative GPCR-like proteins encoded by the $M$. grisea genome rose to 76 when the criteria were relaxed to include homologs of the Pth11 receptor (DeZwaan et al., 1999) which is required for M. grisea pathogenicity in rice. Applying a more universal approach to A. fumigatus, we used the published genome sequence (Nierman et al., 2005) to catalogue all $A$. fumigatus proteins having predicted TMDs (Figure 1). To implement this, we used the TMPRED (Hofmann and Stoffel, 1993) predictive tool to perform an analysis of all 9497 A. fumigatus proteins encoded by the reference genome Af293 (Nierman et al., 2005) http://www.cadre-genomes.org.uk/ Aspergillusfumigatus/Info/Index. In total we identified 6496 proteins having putative TMDs. Among them, 161 proteins were found to encode seven predicted TMDs (Tables 1 and A1). The majority of the predicted 7TMD proteins are of hypothetical function (Table A1).

\begin{tabular}{|c|c|c|c|c|c|c|c|c|}
\hline \multirow[t]{2}{*}{ TMDs } & \multicolumn{8}{|c|}{ Chromosome number } \\
\hline & 1 & 2 & 3 & 4 & 5 & 6 & 7 & 8 \\
\hline 0 & 557 & 519 & 424 & 398 & 428 & 358 & 179 & 138 \\
\hline 1 & 311 & 404 & 378 & 251 & 288 & 282 & 179 & 161 \\
\hline 2 & 267 & 257 & 228 & 199 & 206 & 194 & 106 & 114 \\
\hline 3 & 127 & 233 & 100 & 100 & 141 & 116 & 51 & 57 \\
\hline 4 & 81 & 85 & 59 & 60 & 56 & 57 & 25 & 40 \\
\hline 5 & 132 & 38 & 23 & 3 & 22 & 31 & 18 & 17 \\
\hline 6 & 22 & 26 & 17 & 21 & 16 & 21 & 14 & 8 \\
\hline 7 & 18 & 22 & 23 & 19 & 28 & 27 & 7 & 16 \\
\hline 8 & 9 & 10 & 16 & 9 & 10 & 5 & 5 & 4 \\
\hline 9 & 19 & 13 & 12 & 13 & 15 & 15 & 4 & 9 \\
\hline 10 & 15 & 23 & 20 & 13 & 16 & 7 & 7 & 12 \\
\hline 11 & 22 & 22 & 23 & 29 & 21 & 16 & 9 & 18 \\
\hline 12 & 23 & 25 & 24 & 17 & 18 & 19 & 12 & 9 \\
\hline 13 & 7 & 6 & 9 & 8 & 7 & 8 & 2 & 1 \\
\hline 14 & 3 & 2 & 6 & 3 & 5 & 6 & 2 & 3 \\
\hline 15 & 3 & 1 & 4 & 1 & 2 & 2 & 1 & 1 \\
\hline 16 & 0 & 1 & 2 & 0 & 2 & 4 & 0 & 1 \\
\hline 17 & 0 & 0 & 0 & 0 & 1 & 1 & 0 & 0 \\
\hline 18 & 0 & 0 & 0 & 2 & 1 & 0 & 0 & 0 \\
\hline 19 & 0 & 0 & 0 & 0 & 0 & 0 & 0 & 0 \\
\hline 20 & 0 & 0 & 0 & 0 & 0 & 0 & 0 & 0 \\
\hline 21 & 0 & 0 & 0 & 0 & 0 & 0 & 0 & 0 \\
\hline 22 & 1 & 0 & 0 & 0 & 0 & 0 & 0 & 0 \\
\hline
\end{tabular}

\section{PalH: A PUTATIVE 7TMD pH SENSOR}

During colonization of the mammalian lung A. fumigatus is exposed to a range of microenvironments, of likely differing pHs, not only within the pulmonary niche but also following phagocytosis by macrophages or ingestion by neutrophils and exposure to their vacuole contents (Levitz et al., 1999; Newman, 1999; Reeves et al., 2002; Ibrahim-Granet et al., 2003). Versatility of metabolism and physiology is required to survive such extremes, including appropriate $\mathrm{pH}$-responsive gene expression for nutrient acquisition and survival (Bignell et al., 2005). In the model ascomycete and occasional pathogen $A$. nidulans, the PacC transcription factor governs gene expression in response to extracellular pH (Tilburn et al., 1995; Diez et al., 2002) and is vital for mammalian pathogenicity (Peñalva and Arst, 2004; Bignell et al., 2005; Peñalva et al., 2008). Under alkaline conditions, a signaling cascade involving seven proteins is involved in activation of $\mathrm{PacC}$. A putative $\mathrm{pH}$ sensor, $\mathrm{PalH}$, has 7TMDs and a cytoplasmic C-terminus (Negrete-Urtasun et al., 1997, 1999), which interacts with a cognate arrestin encoded by palF (Herranz et al., 2005; Hervas-Aguilar et al., 2010). Unlike canonical GPCR receptors, PalH is not thought to act via interaction with G-protein subunits (Kroeze et al., 2003). When an alkaline response is triggered, PalF is phosphorylated and subsequently ubiquitinated in a PalH-dependent manner (Herranz et al., 2005), leading to PalB-mediated, signal dependent, proteolytic cleavage of the $\mathrm{pH}$-responsive transcription factor $\mathrm{PacC}$ (Penas et al., 2007; Rodriguez-Galan et al., 2009). Subsequent translocation of the truncated $\mathrm{PacC}$ protein, from cytoplasm to nucleus, permits alkaline adaptation via differential expression of genes required to enable growth under alkaline extracellular conditions (Tilburn et al., 1995; Mingot et al., 1999, 2001; Espeso and Arst, 2000; Espeso et al., 2000). In A. fumigatus the amino acid residues crucial for $\mathrm{PalH}$ and PalF interaction are conserved, and in split-ubiquitin analyses the proteins enter into close proximity (Bertuzzi and Bignell, 2011; Bignell, 2012). We have also recently demonstrated the requirement for A. fumigatus $\mathrm{PalH}$ for murine infection (Bertuzzi et al., in preparation).

\section{HISTIDINE KINASE SENSORS IN A. fumigatus}

Histidine kinases $(\mathrm{HK})$ are phosphorelay protein sensors which transduce extracellular signals. HKs are common in the fungal kingdom, and apparently absent in humans (West and Stock, 2001). Amongst archaea, bacteria and fungi, two classes of HK (two-component and hybrid) are found. The former class of two-component receptor systems predominate in bacteria and archaea, whereby autophosphorylation of the HK protein precedes transfer of the phosphoryl group to a conserved aspartate residue in a second protein, termed the response regulator (RR)(Li et al., 2012). HK activities have been associated with both the osmo- and peroxide-regulatory pathways in multiple fungi, and have been most extensively characterized in S. cerevisiae (Santos and Shiozaki, 2001). However, RR proteins are not abundantly encoded by fungal genomes; Skn7 and Ssk1 are two examples of such proteins, which in S. cerevisiae and C. albicans, account for the entire RR cohort of these species (Kaserer et al., 2009; Oide et al., 2010). The fungal phosphotransfer relay can involve three proteins, as exemplified by the S. cerevisiae HOG1 
MAPK phosphorelay, where an HK (Sln 1$)$, a histidine phosphointermediate (Ypd1) and an RR (Ssk1) collectively mediate a multistep phosphotransfer (Kaserer et al., 2009).

Fungal HKs most commonly fall into the hybrid class of regulators which utilize a single polypeptide. This protein possesses both a Histidine kinase A (HiskA) and a receiver domain (REC) containing a conserved aspartate residue (Li et al., 2012). Other domains, such as the ATP-binding HATPase_c domain (Dago et al., 2012) are also found; however, as these proteins are largely uncharacterized for A. fumigatus, the functional relevance of domain organization is unknown. The composition, and/or relative positioning, of additional domains provides the basis for sub-classification of HKs (Catlett et al., 2003), presented for 12 A. fumigatus HKs in Table A2. Amongst these, only three have been studied: the two-component system proteins $\mathrm{A}, \mathrm{B}$, and $\mathrm{C}$ (TcsA/Fos-1 AFUA_5G10240, TcsB AFUA_2G00660 and TcsC AFUA_2G03560).

Despite the significance of the HK Fos-1 for detection of extracellular stresses, this hybrid HK has been previously predicted as possessing no TMDs, implying a cytosolic presence (Pott et al., 2000). However, our TMPRED analyses predicted TMDs for all of the three HKs, with Fos-1 possessing a single TMD (Table A2). Deletion of the fos -1 gene leads to a $\sim 66 \%$ reduction in conidiation after $48 \mathrm{~h}$ in liquid YG medium, as well as heightened resistance to the cell wall-degrading enzyme mix novozym 234, suggesting the role of fos- 1 in cell wall assembly (Pott et al., 2000). $\Delta$ fos- 1 mutants were found to have normal morphology, germination, osmotic and oxidative stress tolerance, and antifungal susceptibilities. Subsequent transcriptional analyses found a significant increase in fos- 1 expression, relative to in vitro growth, during the first $72 \mathrm{~h}$ of infection in a murine model of pulmonary aspergillosis (Zhang et al., 2005), and reduced virulence of $A$. fumigatus in a systemic murine model of infection (Clemons et al., 2002).

In a study addressing the role of oxidative stress in A. fumigatus pathogenicity, Du et al. (2006) characterized the A. fumigatus TcsB protein, a putative homolog of $\operatorname{Sn} 1$ in $S$. cerevisiae. In A. nidulans, TMPRED analysis predicted 2 TMDs for TcsB at the N-terminus (Furukawa et al., 2002), though in A. fumigatus, our prediction extends this to 4TMDs (Table A1). Unlike S. cerevisiae where deletion of $\sin 1$ is lethal (Maeda et al., 1994), an A. fumigatus $\Delta t c s B$ mutant is viable, demonstrates normal morphology, and is as tolerant as the wild type to increased temperatures, various cell wall damaging agents, and poor nitrogen/carbon sources. The only phenotype discernable for the mutant was a minor sensitivity to SDS (Du et al., 2006). This data suggests a nonessential role for $\mathrm{TcsB}$, or redundancy of function with other, as yet uncharacterized protein(s).

It is believed that group III HK mediate resistance to high osmotic pressure via the high osmolarity glycerol pathway (HOG). For this reason, the characterization of the sole A. fumigatus group III hybrid HK TcsC, classified as such on the basis of putative HAMP (HK, adenyl cyclase, methylaccepting chemotaxis protein, phosphatase) domains, was investigated (McCormick et al., 2012). The significance of the HAMP domains, based upon studies of other sensor proteins and signaling is postulated as providing the means to couple input and output since HAMP domains of integral membrane hybrid
HKs are found in close proximity to the membrane-spanning segment (Parkinson, 2010). It is speculated that in response to extracellular signals, such as altered osmolarity, a conformational rearrangement is triggered which prompts activation of an output domain (Parkinson, 2010). In A. fumigatus, deletion of the tcs $C$ gene resulted in an extended white colony rim and a reduced number of extending hyphae. However, unlike the A. nidulans homologue nikA (Hagiwara et al., 2009), no detrimental effects on sporulation and conidial growth were observed. In the presence of nitrate as a nitrogen source a significant reduction in radial growth was detectable, and furthermore, compared to the control strain, growth of $\Delta t c s C$ at $2 \% \mathrm{O}_{2}$ abolished sporulation and prompted a dome-shaped morphology indicative of oxygen starvation. A strong inhibition of growth resulted from exposure to hyperosmotic stress $(1.2 \mathrm{M}$ sorbitol, $1 \mathrm{M} \mathrm{KCl}$, or $1 \mathrm{M} \mathrm{NaCl}$ ) but sensitivity to calcofluor white, amphotericin $\mathrm{B}$, posaconazole and caspofungin, extremes of $\mathrm{pH} /$ temperature, or oxidative stress were reportedly normal.

In a comparative analysis of wild type and $\Delta t c s C$ virulence, no discernable differences in pathogenicity analysis in a murine model of invasive aspergillosis were detected (McCormick et al., 2012).

\section{CELL WALL RECEPTORS}

The fungal cell wall is essential for viability and an important target of antifungal drugs (Latgé et al., 2005; Latgé, 2007; Walker et al., 2010). In fungi a conserved MAPK signaling module is responsible for maintaining cellular integrity, shape and resilience to environmental stresses (Lee and Levin, 1992; Levin, 2005; Lesage and Bussey, 2006). In S. cerevisiae, such MAPK signaling (Chen and Thorner, 2007) is initiated through stress detection at five integral membrane receptors Wsc1-3, Mid2, and Mtl1 (Lodder et al., 1999). This promotes guanine nucleotide exchange factor (GEFs-Rom1 and Rom2)-mediated nucleotide exchange upon the GTPase Rho1, facilitating the regulation of numerous downstream effectors ( $\mathrm{Zu}$ et al., 2001). In a quest to find equivalent cell wall sensors in A. fumigatus, Dichtl et al. (2012) performed BLAST analyses to reveal three previously uncharacterized open reading frames with domain structures similar to those of Wsc1-3 (Af Wsc1, AFUA_4G13670, Af Wsc2, AFUA_3G07050, and Af Wsc3, AFUA_5G09020 respectively). Bioinformatic analyses predicted the presence of characteristic cell wall integrity (CWI) sensor N-terminal WSC domains with downstream, though truncated, ser/thr rich regions, and (with the exception of Wsc2) transmembrane domains. In common with the $S$. cerevisiae sensors a short cytosolic C-terminus was also predicted for two of the sensors (Dichtl et al., 2012).

To discern subcellular localization, ectopically integrating vectors were applied to generate four putative CWI sensor-GFP fusions, Wsc1-3, and MidA. From these, localization of all C-terminally tagged sensors was observed at the fungal surface. Additionally a strong presence was observed in vacuoles, though this was dismissed as a by-product of over expression or misfolding. Phenotypic analyses of single and double mutants identified a significant impairment of radial growth in the case of a $\Delta w s c 1 \Delta w s c 3$ double mutant. These findings were further exacerbated in a triple mutant $\Delta w s c 1 \Delta w s c 3 \Delta$ midA. Furthermore, in all mutants lacking $w s c 1$, provision of glycerol as 
carbon source lead to a significant reduction in radial growth on minimal media (Dichtl et al., 2012).

Previously, mutants lacking members of the CWI MAPK pathway have demonstrated a clear sensitivity to echinocandins and azole antifungals (Fujioka et al., 2007; Dirr et al., 2010). Extending this analysis to the A. fumigatus mutant phenotypes revealed a single relevant phenotype, namely the heightened sensitivity of the $\Delta w s c 1$ mutant to the echinocandin, caspofungin (Dichtl et al., 2012).

To study stress-induced activation of intracellular signaling, effects on growth and MpkA phosphorylation were analyzed in the presence of the cell wall perturbing agent, calcoflour white, or following heat shock $\left(48^{\circ} \mathrm{C}\right)$. None of the Wsc mutants were found to be sensitive to cell wall perturbation or heat shock, however, $\triangle$ midA was highly sensitive to all of these stresses. In agreement with phenotypic data, calcofluor white-induced MpkA phosphorylation was significantly reduced in the $\Delta$ midA mutant compared with wild type, while phosphorylation of MpkA was not diminished in mutants lacking $w s c 1$ or $w s c 1$ and $w s c 3$ (Dichtl et al., 2012). In S. cerevisiae, the Wsc1 cell wall sensor mediates signaling of alkaline stress via the CWI MAPK module (Serrano et al., 2006); no evidence for such a role in A. fumigatus was obtained. Thus, while Mkk2 null mutants are sensitive to alkalinization of the medium (Dirr et al., 2010), the identity of the activating cell wall sensor remains unknown.

Taken together these findings suggest that MidA is the sole cell wall perturbation sensor, while Wscl is required for glycerol carbon source assimilation. Furthermore, a compensatory role between Wscl and Wsc3 with regards to efficient growth and conidiation has been demonstrated. Despite these observations, a role for Wsc2 has yet to be identified, while the putative CWI pH sensor remains elusive.

\section{RECEPTOR-MEDIATED SIGNALING DURING A. fumigatus INFECTION: RELEVANCE FOR THERAPEUTIC STRATEGY}

Drugs which target GPCR function account for $>50 \%$ of currently licensed drugs (Davies et al., 2008). It therefore follows

\section{REFERENCES}

Affeldt, K. J., Brodhagen, M., and Keller, N. P. (2012). Aspergillus oxylipin signaling and quorum sensing pathways depend on G protein-coupled receptors. Toxins (Basel) 4, 695-717.

Balloy, V., and Chignard, M. (2009). The innate immune response to Aspergillus fumigatus. Microbes Infect. 11, 919-927.

Bertuzzi, M., and Bignell, E. M. (2011). Sensory perception in fungal pathogens: applications of the split-ubiquitin Membrane Yeast Two-Hybrid (MYTH) technique. Fungal Biol. Rev. 25, 7-7.

Bignell, E. (2012). Conservation of seven transmembrane domain receptors and arrestins required for $\mathrm{pH}$ signalling in Aspergillus fumigatus, and implications for drug discovery. Ann. N.Y. Acad. Sci. 1273, 35-43.

Bignell, E., Negrete-Urtasun, S., Calcagno, A. M., Haynes, K., Arst, H. N. Jr., and Rogers, T. (2005). The Aspergillus $\mathrm{pH}$-responsive transcription factor $\mathrm{PacC}$ regulates virulence. Mol. Microbiol. 55, 1072-1084.

Casadevall, A., Steenbergen, J. N., and Nosanchuk, J. D. (2003). 'Ready made' virulence and 'dual use' virulence factors in pathogenic environmental fungi-the Cryptococcus neoformans paradigm. Curr. Opin. Microbiol. 6, 332-337.

Catlett, N. L., Yoder, O. C., and Turgeon, B. G. (2003). Wholegenome analysis of two-component signal transduction genes in fungal pathogens. Eukaryot. Cell 2, 1151-1161.

that fungal GPCRs are likely to be similarly responsive to chemical perturbations. This fact, coupled with the absolute requirement for some GPCRs in fungal growth make a compelling case for these proteins as antifungal drug targets. Although the pharmaceutical market is dominated by GPCR-active molecules, the discovery of most of these agents was made on the basis of functional activity in high throughput screens, only later were the targets and modes of action clarified (Filmore, 2004). In the post-genomic era, with confidence in the pharmaceutical relevance of such proteins, drug discovery can become targetdriven. An expanding repertoire of technologies to probe 7TMD protein activities provides the basis upon which to confront functional studies of the uncharacterized receptors in A. fumigatus and to screen for inhibitory molecules. It has recently been suggested that considering seven transmembrane receptors as disordered proteins able to allosterically respond to a number of binding partners, is useful in understanding the plasticity of function exhibited by such proteins (Kenakin and Miller, 2010). Conformational changes which occur in response to extracellular ligands and/or stimuli expose cytosolic signaling domains and present three distinct arenas open to perturbation: extracellular sensing/ligand binding, cytosolic surfaces, and intramembrane domains. In order to prioritise the most promising candidates for drug development, a crucial experiment will be to assess the requirement of such receptors, via regulatable promoters, for sustained viability of established fungal mass in murine models of infection (Gossen and Bujard, 1992).

\section{ACKNOWLEDGMENTS}

We gratefully acknowledge the assistance of Arshad Khan in automating the genome-wide TMPRED analyses. This work was assisted by funding from the Medical Research Council [G0501164 to E. M. Bignell], the Biotechnology and Biological Sciences Research Council [PhD studentship to C. M. Grice, BB/F016239/1], and the Wellcome Trust [WT093596MA to E. M. Bignell].

Chamilos, G., Luna, M., Lewis, R. E., Bodey, G. P., Chemaly, R., Tarrand, J. J., et al. (2006). Invasive fungal infections in patients with hematologic malignancies in a tertiary care cancer center: an autopsy study over a 15-year period (1989-2003). Haematologica 91, 986-989.

Chen, R. E., and Thorner, J. (2007). Function and regulation in MAPK signaling pathways: lessons learned from the yeast Saccharomyces cerevisiae. Biochim. Biophys. Acta 1773, 1311-1340.

Clemons, K. V., Miller, T. K., Selitrennikoff, C. P., and Stevens, D. A. (2002). fos-1, a putative histidine kinase as a virulence factor for systemic aspergillosis. Med. Mycol. 40, 259-262.

Dagenais, T. R., and Keller, N. P. (2009). Pathogenesis of Aspergillus fumigatus in invasive Aspergillosis. Clin. Microbiol. Rev. 22, 447-465.

Dago, A. E., Schug, A., Procaccini, A., Hoch, J. A., Weigt, M., and Szurmant, H. (2012). Structural basis of histidine kinase autophosphorylation deduced by integrating genomics, molecular dynamics, and mutagenesis. Proc. Natl. Acad. Sci. U.S.A. 109, E1733-E1742.

Davies, M. N., Secker, A., HallingBrown, M., Moss, D. S., Freitas, A. A., Timmis, J., et al. (2008). GPCRTree: online hierarchical classification of GPCR function. BMC Res. Notes 1:67. doi: 10.1186/17560500-1-67

DeZwaan, T. M., Carroll, A. M., Valent, B., and Sweigard, J. A. (1999). Magnaporthe grisea Pthllp is a novel plasma membrane protein 
that mediates appressorium differentiation in response to inductive substrate cues. Plant Cell 11, 2013-2030.

Dichtl, K., Helmschrott, C., Dirr, F., and Wagener, J. (2012). Deciphering cell wall integrity signalling in Aspergillus fumigatus: identification and functional characterization of cell wall stress sensors and relevant Rho GTPases. Mol. Microbiol. 83, 506-519.

Diez, E., Alvaro, J., Espeso, E. A., Rainbow, L., Suarez, T., Tilburn, J., et al. (2002). Activation of the Aspergillus PacC zinc finger transcription factor requires two proteolytic steps. EMBO J. 21, 1350-1359.

Dirr, F., Echtenacher, B., Heesemann, J., Hoffmann, P., Ebel, F., and Wagener, J. (2010). AfMkk2 is required for cell wall integrity signaling, adhesion, and full virulence of the human pathogen Aspergillus fumigatus. Int. J. Med. Microbiol. 300, 496-502.

Du, C., Sarfati, J., Latge, J. P., and Calderone, R. (2006). The role of the sakA $(H o g 1)$ and $t c s B(\sin 1)$ genes in the oxidant adaptation of Aspergillus fumigatus. Med. Mycol. 44, 211-218.

Espeso, E., and Arst, H. N. Jr. (2000). On the mechanism by which alkaline $\mathrm{pH}$ prevents expression of an acid-expressed gene. Mol. Cell. Biol. 20, 3355.

Espeso, E., Roncal, T., Díez, E., Rainbow, L., Bignell, E., Álvaro, J., et al. (2000). On how a transcription factor can avoid its proteolytic activation in the absence of signal transduction. EMBO J. 19, Vol. 7 719-728.

Filmore, D. (2004). It's a GPCR World. Mod. Drug Discov. 7, 24-28.

Fujioka, T., Mizutani, O., Furukawa, K., Sato, N., Yoshimi, A., Yamagata, Y., et al. (2007). MpkA-Dependent and -independent cell wall integrity signaling in Aspergillus nidulans. Eukaryot. Cell 6, 1497-1510.

Furukawa, K., Katsuno, Y., Urao, T., Yabe, T., Yamada-Okabe, Y., Yamada-Okabe, H., et al. (2002). Isolation and functional analysis of a gene, $t c s B$, encoding a transmembrane hybrid-type histidine kinase from Aspergillus nidulans. Appl. Environ. Microbiol. 68, 5304-5310.

Gehrke, A., Heinekamp, T., Jacobsen, I. D., and Brakhage, A. A. (2010). Heptahelical receptors GprC and $\mathrm{GprD}$ of Aspergillus fumigatus are essential regulators of colony growth, hyphal morphogenesis, and virulence. Appl. Environ. Microbiol. 76, 3989-3998.

Gossen, M., and Bujard, H. (1992). Tight control of gene expression in mammalian cells by tetracyclineresponsive promoters. Proc. Natl. Acad. Sci. U.S.A. 89, 5547-5551.

Hagiwara, D., Mizuno, T., and Abe, K. (2009). Characterization of NikA histidine kinase and two response regulators with special reference to osmotic adaptation and asexual development in Aspergillus nidulans. Biosci. Biotechnol. Biochem. 73, 1566-1571.

Herranz, S., Rodriguez, J. M., Bussink, H. J., Sanchez-Ferrero, J. C., Arst, H. N. Jr., Penalva, M. A., et al. (2005). Arrestin-related proteins mediate $\mathrm{pH}$ signaling in fungi. Proc. Natl. Acad. Sci. U.S.A. 102, 12141-12146.

Hervas-Aguilar, A., Galindo, A., and Penalva, M. A. (2010). Receptorindependent Ambient $\mathrm{pH}$ signaling by ubiquitin attachment to fungal arrestin-like PalF. J. Biol. Chem. 285, 18095-18102.

Hofmann, K., and Stoffel, W. (1993). TMbase - a database of membrane spanning protein segments. Biol. Chem. 374, 166.

Horn, F., Bettler, E., Oliveira, L., Campagne, F., Cohen, F. E., and Vriend, G. (2003). GPCRDB information system for $G$ proteincoupled receptors. Nucleic Acids Res. 31, 294-297.

Ibrahim-Granet, O., Philippe, B., Boleti, H., Boisvieux-Ulrich, E., Grenet, D., Stern, M., et al. (2003). Phagocytosis and intracellular fate of Aspergillus fumigatus conidia in alveolar macrophages. Infect. Immun. 71, 891-903.

Karpichev, I. V., Cornivelli, L., and Small, G. M. (2002). Multiple regulatory roles of a novel Saccharomyces cerevisiae protein, encoded by YOL002c, in lipid and phosphate metabolism. J. Biol. Chem. 277, 19609-19617.

Kaserer, A. O., Andi, B., Cook, P. F., and West, A. H. (2009). Effects of osmolytes on the SLN1YPD1-SSK1 phosphorelay system from Saccharomyces cerevisiae. Biochemistry 48, 8044-8050.

Kenakin, T., and Miller, L. J. (2010). Seven transmembrane receptors as shapeshifting proteins: the impact of allosteric modulation and functional selectivity on new drug discovery. Pharmacol. Rev. 62, 265-304.

Kraakman, L., Lemaire, K., Ma, P., Teunissen, A. W., Donaton, M. C., Van Dijck, P., et al. (1999). A Saccharomyces cerevisiae G-protein coupled receptor, Gpr1, is specifically required for glucose activation of the cAMP pathway during the transition to growth on glucose. Mol. Microbiol. 32, 1002-1012.
Kroeze, W. K., Sheffler, D. J., and Roth, B. L. (2003). G-protein-coupled receptors at a glance. J. Cell Sci. 116, 4867-4869.

Kulkarni, R. D., Thon, M. R., Pan, H., and Dean, R. A. (2005). Novel Gprotein-coupled receptor-like proteins in the plant pathogenic fungus Magnaporthe grisea. Genome Biol. 6:R24. doi: 10.1186/gb-2005-63-r24

Lafon, A., Han, K. H., Seo, J. A., Yu, J. H., and d'Enfert, C. (2006). Gprotein and cAMP-mediated signaling in aspergilli: a genomic perspective. Fungal Genet. Biol. 43, 490-502.

Latgé, J.-P. (2007). The cell wall: a carbohydrate armour for the fungal cell. Mol. Microbiol. 66, 279-290.

Latgé, J. P., Mouyna, I., Tekaia, F., Beauvais, A., Debeaupuis, J. P., and Nierman, W. (2005). Specific molecular features in the organization and biosynthesis of the cell wall of Aspergillus fumigatus. Med. Mycol. 43(Suppl. 1),15-22.

Lee, K. S., and Levin, D. E. (1992). Dominant mutations in a gene encoding a putative protein kinase (BCK1) bypass the requirement for a Saccharomyces cerevisiae protein kinase C homolog. Mol. Cell. Biol. $12,172-182$.

Lesage, G., and Bussey, H. (2006). Cell wall assembly in Saccharomyces cerevisiae. Microbiol. Mol. Biol. Rev. 70, 317-343.

Levin, D. E. (2005). Cell wall integrity signaling in Saccharomyces cerevisiae. Microbiol. Mol. Biol. Rev. 69 , 262-291.

Levitz, S. M., Nong, S. H., Seetoo K. F., Harrison, T. S., Speizer, R. A., and Simons, E. R. (1999). Cryptococcus neoformans resides in an acidic phagolysosome of human macrophages. Infect. Immun. 67, 885-890.

Li, D., Agrellos, O. A., and Calderone, R. (2012). Histidine kinases keep fungi safe and vigorous. Curr. Opin. Microbiol. 13, 424-430.

Liebmann, B., Gattung, S., Jahn, B., and Brakhage, A. A. (2003). cAMP signaling in Aspergillus fumigatus is involved in the regulation of the virulence gene pksP and in defense against killing by macrophages. Mol. Genet. Genomics 269, 420-435.

Lodder, A. L., Lee, T. K., and Ballester, R. (1999). Characterization of the Wscl protein, a putative receptor in the stress response of Saccharomyces cerevisiae. Genetics 152, 1487-1499.

Lyons, T. J., Villa, N. Y., Regalla, L. M., Kupchak, B. R., Vagstad, A., and Eide, D. J. (2004). Metalloregulation of yeast membrane steroid receptor homologs. Proc. Natl. Acad. Sci. U.S.A. 101, 5506-5511.

Maeda, T., Wurgler-Murphy, S. M., and Saito, H. (1994). A two-component system that regulates an osmosensing MAP kinase cascade in yeast. Nature 369, 242-245.

McCormick, A., Jacobsen, I. D., Broniszewska, M., Beck, J., Heesemann, J., and Ebel, F. (2012). The two-component sensor kinase $\mathrm{Tcs} C$ and its role in stress resistance of the humanpathogenic mold Aspergillus fumigatus. PLoS ONE 7:e38262. doi: 10.1371/journal.pone.0038262

McNeil, M. M., Nash, S. L., Hajjeh, R. A., Phelan, M. A., Conn, L. A., Plikaytis, B. D., et al. (2001). Trends in mortality due to invasive mycotic diseases in the United States, 1980-1997. Clin. Infect. Dis. 33, 641-647.

Miller, T. L., Godfrey, P. A., Dealmeida, V. I., and Mayo, K. E. (1999). The rat growth hormone-releasing hormone receptor gene: structure, regulation, and generation of receptor isoforms with different signaling properties. Endocrinology 140, 4152-4165.

Mingot, J. M., Espeso, E. A., Diez, E., and Penalva, M. A. (2001). Ambient $\mathrm{pH}$ signaling regulates nuclear localization of the Aspergillus nidulans PacC transcription factor. Mol. Cell. Biol. 21, 1688-1699.

Mingot, J. M., Tilburn, J., Diez, E., Bignell, E., Orejas, M., Widdick, D. A., et al. (1999). Specificity determinants of proteolytic processing of Aspergillus PacC transcription factor are remote from the processing site, and processing occurs in yeast if $\mathrm{pH}$ signalling is bypassed. Mol. Cell. Biol. 19, 1390-1400.

Negrete-Urtasun, S., Denison, S. H., and Arst, H. N. Jr. (1997). Characterization of the $\mathrm{pH}$ signal transduction pathway gene palA of Aspergillus nidulans and identification of possible homologs. J. Bacteriol. 179, 1832-1835.

Negrete-Urtasun, S., Reiter, W., Diez, E., Denison, S. H., Tilburn, J., Espeso, E. A., et al. (1999). Ambient $\mathrm{pH}$ signal transduction in Aspergillus: completion of gene characterization. Mol. Microbiol. 33, 994-1003.

Newman, S. L. (1999). Macrophages in host defense against Histoplasma capsulatum. Trends Microbiol. 7, 67-71.

Nierman, W. C., Pain, A., Anderson, M. J., Wortman, J. R., Kim, H. S., Arroyo, J., et al. (2005). Genomic sequence of the pathogenic and allergenic filamentous fungus 
Aspergillus fumigatus. Nature 438, 1151-1156.

Oide, S., Liu, J. Y., Yun, S. H., Wu, D. L., Michev, A., Choi, M. Y., et al. (2010). Histidine kinase two-component response regulator proteins regulate reproductive development, virulence, and stress responses of the fungal cereal pathogens Cochliobolus heterostrophus and Gibberella zeae. Eukaryot. Cell 9, 1867-1880.

Parkinson, J. S. (2010). Signaling mechanisms of HAMP domains in chemoreceptors and sensor kinases. Annu. Rev. Microbiol. 64, 101-122.

Peñalva, M., and Arst, H. N. Jr. (2004). Recent advances in the characterization of ambient $\mathrm{pH}$ regulation of gene expression in filamentous fungi and yeasts. Аnnu. Rev. Microbiol. 58, 425-451.

Peñalva, M., Tilburn, J., Bignell, E., and Arst H. N. Jr. (2008). Ambient pH gene regulation in fungi: making connections. Trends Microbiol. 16, 291-300.

Penas, M. M., Hervas-Aguilar, A., Munera-Huertas, T., Reoyo, E., Penalva, M. A., Arst, H. N. Jr., et al. (2007). Further characterization of the signaling proteolysis step in the Aspergillus nidulans $\mathrm{pH}$ signal transduction pathway. Eukaryot. Cell 6, 960-970.

Pott, G. B., Miller, T. K., Bartlett, J. A., Palas, J. S., and Selitrennikoff, C. P. (2000). The isolation of FOS-1, a gene encoding a putative two-component histidine kinase from Aspergillus fumigatus. Fungal Genet. Biol. 31, 55-67.

Reeves, E. P., Lu, H., Jacobs, H. L. Messina, C. G., Bolsover, S., Gabella, G., et al. (2002). Killing activity of neutrophils is mediated through activation of proteases by $\mathrm{K}^{+}$flux. Nature 416, 291-297.

Rhodes, J. C. (2006). Aspergillus fumigatus: growth and virulence. Med. Mycol. 44(Suppl. 1), S77-S81.

Rodriguez-Galan, O., Galindo, A., Hervas-Aguilar, A., Arst, H. N. Jr., and Penalva, M. A. (2009). Physiological involvement in $\mathrm{pH}$ signaling of Vps24-mediated recruitment of Aspergillus PalB cysteine protease to ESCRT-III. J. Biol. Chem. 284, 4404-4412.

Santos, J. L., and Shiozaki, K. (2001). Fungal histidine kinases. Sci. STKE 2001:re1. doi: 10.1126/stke. 2001.98.re1

Schrettl, M., Bignell, E., Kragl, C., Joechl, C., Rogers, T., Arst, H. N. Jr., et al. (2004). Siderophore biosynthesis but not reductive iron assimilation is essential for Aspergillus fumigatus virulence. J. Exp. Med. 200, 1213-1219.

Schrettl, M., Bignell, E., Kragl, C., Sabiha, Y., Loss, O., Eisendle, M., et al. (2007). Distinct roles for intraand extracellular siderophores during Aspergillus fumigatus infection. PLoS Pathog. 3:1195-1207. doi: 10.1371/journal.ppat.0030128

Serrano, R., Martin, H., Casamayor, A., and Arino, J. (2006). Signaling alkaline $\mathrm{pH}$ stress in the yeast Saccharomyces cerevisiae through the Wscl cell surface sensor and the Slt2 MAPK pathway. J. Biol. Chem. 281, 39785-39795.

Shin, K. S., Kwon, N. J., and Yu, J. H. (2009). Gbg-mediated growth and developmental control in Aspergillus fumigatus. Curr. Genet. 55, 631-641.

Tilburn, J., Sarkar, S., Widdick, D. A., Espeso, E. A., Orejas, M., Mungroo, J., et al. (1995). The Aspergillus $\mathrm{PacC}$ zinc finger transcription factor mediates regulation of both acid- and alkaline-expressed genes by ambient pH. EMBO J. 14, 779-790.

Walker, L. A., Gow, N. A. R., and Munro, C. A. (2010). Fungal echinocandin resistance. Fungal Genet. Biol. 47, 117-126.

West, A. H., and Stock, A. M. (2001). Histidine kinases and response regulator proteins in two-component signaling systems. Trends Biochem. Sci. 26, 369-376.

Yun, C. W., Tamaki, H., Nakayama, R., Yamamoto, K., and Kumagai, $\mathrm{H}$ (1998). Gprlp, a putative G-protein coupled receptor, regulates glucosedependent cellular cAMP level in yeast Saccharomyces cerevisiae. Biochem. Biophys. Res. Commun. 252, 29-33.

Zhang, L. J., Wang, M. Y., Li, R. Y., and Calderone, R. (2005). Expression of Aspergillus fumigatus virulence-related genes detected in vitro and in vivo with competitive
RT-PCR. Mycopathologia 160, 201-206.

$\mathrm{Zu}, \mathrm{T}$, Verna, J., and Ballester, R. (2001). Mutations in WSC genes for putative stress receptors result in sensitivity to multiple stress conditions and impairment of Rlm1-dependent gene expression in Saccharomyces cerevisiae. Mol. Genet. Genomics 266, 142-155.

Conflict of Interest Statement: The authors declare that the research was conducted in the absence of any commercial or financial relationships that could be construed as a potential conflict of interest.

Received: 08 November 2012; accepted: 01 February 2013; published online: 20 February 2013.

Citation: Grice CM, Bertuzzi $M$ and Bignell EM (2013) Receptor-mediated signaling in Aspergillus fumigatus. Front. Microbio. 4:26. doi: 10.3389/ fmicb.2013.00026

This article was submitted to Frontiers in Fungi and Their Interactions, a specialty of Frontiers in Microbiology.

Copyright (c) 2013 Grice, Bertuzzi and Bignell. This is an open-access article distributed under the terms of the Creative Commons Attribution License, which permits use, distribution and reproduction in other forums, provided the original authors and source are credited and subject to any copyright notices concerning any third-party graphics etc. 


\section{APPENDIX}

Table A1 | Identity and annotations of $A$. fumigatus proteins having seven predicted transmembrane domains.

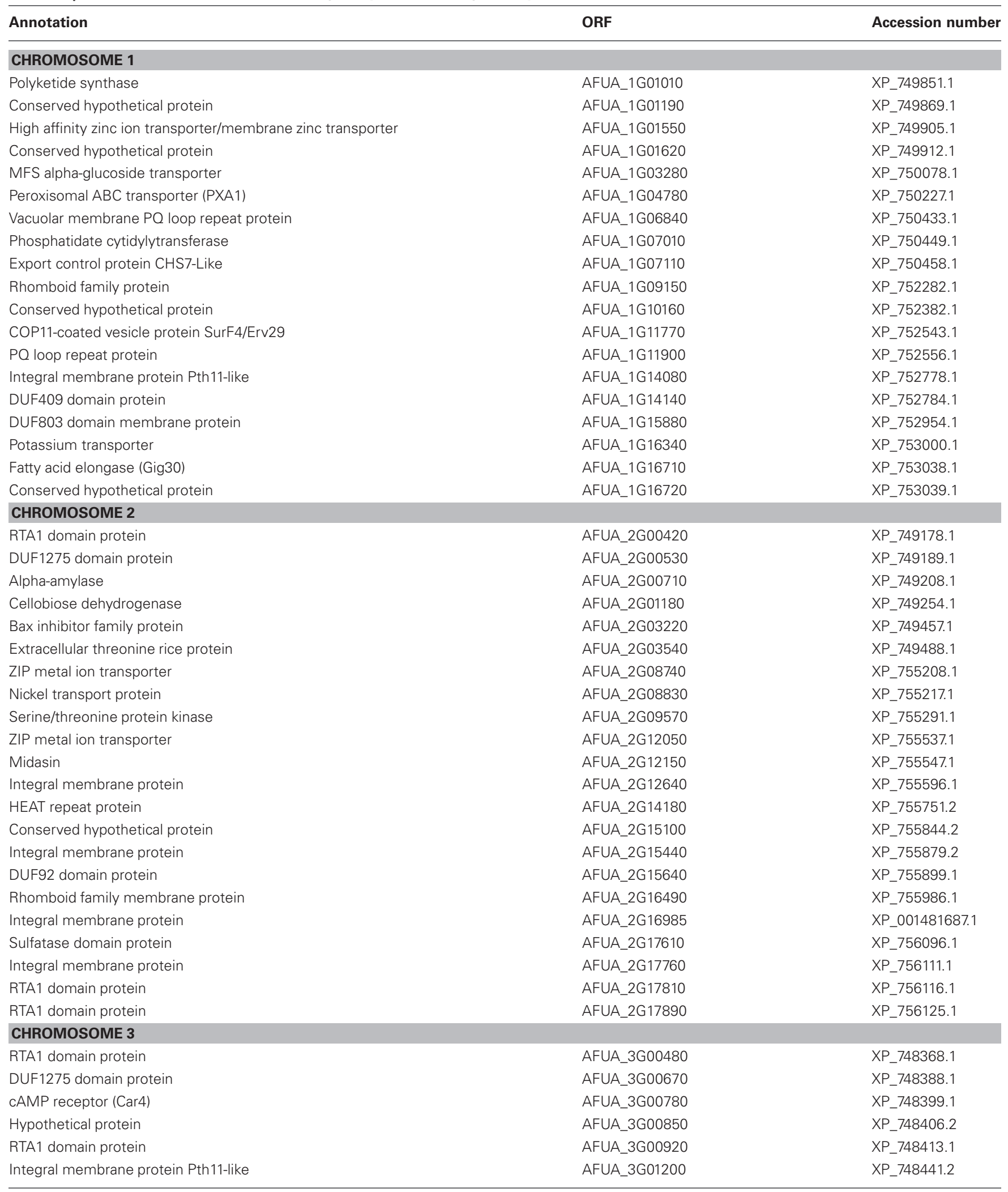


Table A1 | Continued

\begin{tabular}{|c|c|c|}
\hline Annotation & ORF & Accession number \\
\hline G protein coupled receptor family protein & AFUA_3G01750 & XP_748496.1 \\
\hline RTA1 domain protein & AFUA_3G03310 & XP_748650.1 \\
\hline PKS-like enzyme & AFUA_3G03540 & XP_748674.1 \\
\hline UPF0016 domain protein & AFUA_3G07080 & XP_754898.1 \\
\hline Conserved hypothetical protein & AFUA_3G07420 & XP_754867.2 \\
\hline DUF1275 domain protein & AFUA_3G07550 & XP_754856.1 \\
\hline Sucrose transporter & AFUA_3G08480 & XP_754766.1 \\
\hline Conserved hypothetical protein & AFUA_3G09650 & XP_754654.1 \\
\hline $\mathrm{PQ}$ loop repeat protein & AFUA_3G10470 & XP_754572.1 \\
\hline Conserved hypothetical protein & AFUA_3G14870 & XP_754138.1 \\
\hline Integral membrane protein & AFUA_3G15100 & XP_754114.2 \\
\hline \multicolumn{3}{|l|}{ CHROMOSOME 4} \\
\hline Polyketide synthase & AFUA_4G00210 & XP_746435.1 \\
\hline Hypothetical protein & AFUA_4G00580 & XP_746398.1 \\
\hline Hypothetical protein & AFUA_4G01242 & XP_746333.2 \\
\hline Conserved hypothetical protein & AFUA_4G01350 & XP_746323.2 \\
\hline Patatin-like serine hydrolase & AFUA_4G03000 & XP_746486.1 \\
\hline Aquaporin & AFUA_4G03390 & XP_746526.2 \\
\hline Integral membrane protein & AFUA_4G03540 & XP_746541.1 \\
\hline C4-dicarboxylate transporter/malic acid transport protein, putative & AFUA_4G04540 & XP_746640.1 \\
\hline Endosomal peripheral membrane protein (Mon2) & AFUA_4G12070 & XP_751624.1 \\
\hline Potassium uptake transporter & AFUA_4G13540 & XP_751477.1 \\
\hline Conserved hypothetical protein & AFUA_4G14210 & XP_751411.1 \\
\hline Low affinity iron transporter & AFUA_4G14640 & XP_751369.1 \\
\hline \multicolumn{3}{|l|}{ CHROMOSOME 5} \\
\hline Integral membrane protein & AFUA_5G00100 & XP_748330.2 \\
\hline RTA1 domain protein & AFUA_5G01230 & XP_748219.1 \\
\hline RTA1 domain protein & AFUA_5G01310 & XP_748211.1 \\
\hline Phosphate permease & AFUA_5G01320 & XP_748210.1 \\
\hline Histone acetylase complex subunit Paf400 & AFUA_5G02570 & XP_748085.1 \\
\hline Integral membrane protein & AFUA_5G02860 & XP_748057.2 \\
\hline PQ loop repeat protein & AFUA_5G04100 & XP_747934.1 \\
\hline cAMP receptor-like protein & AFUA_5G04135 & XP_001481495.1 \\
\hline Conserved hypothetical protein & AFUA_5G06570 & XP_753976.1 \\
\hline Integral membrane protein & AFUA_5G06670 & XP_753966.1 \\
\hline DUF300 domain protein & AFUA_5G07250 & XP_753909.1 \\
\hline a-pheromone receptor PreA & AFUA_5G07880 & XP_753848.1 \\
\hline $\mathrm{PQ}$ loop repeat protein & AFUA_5G08410 & XP_753796.1 \\
\hline
\end{tabular}


Table A1 | Continued

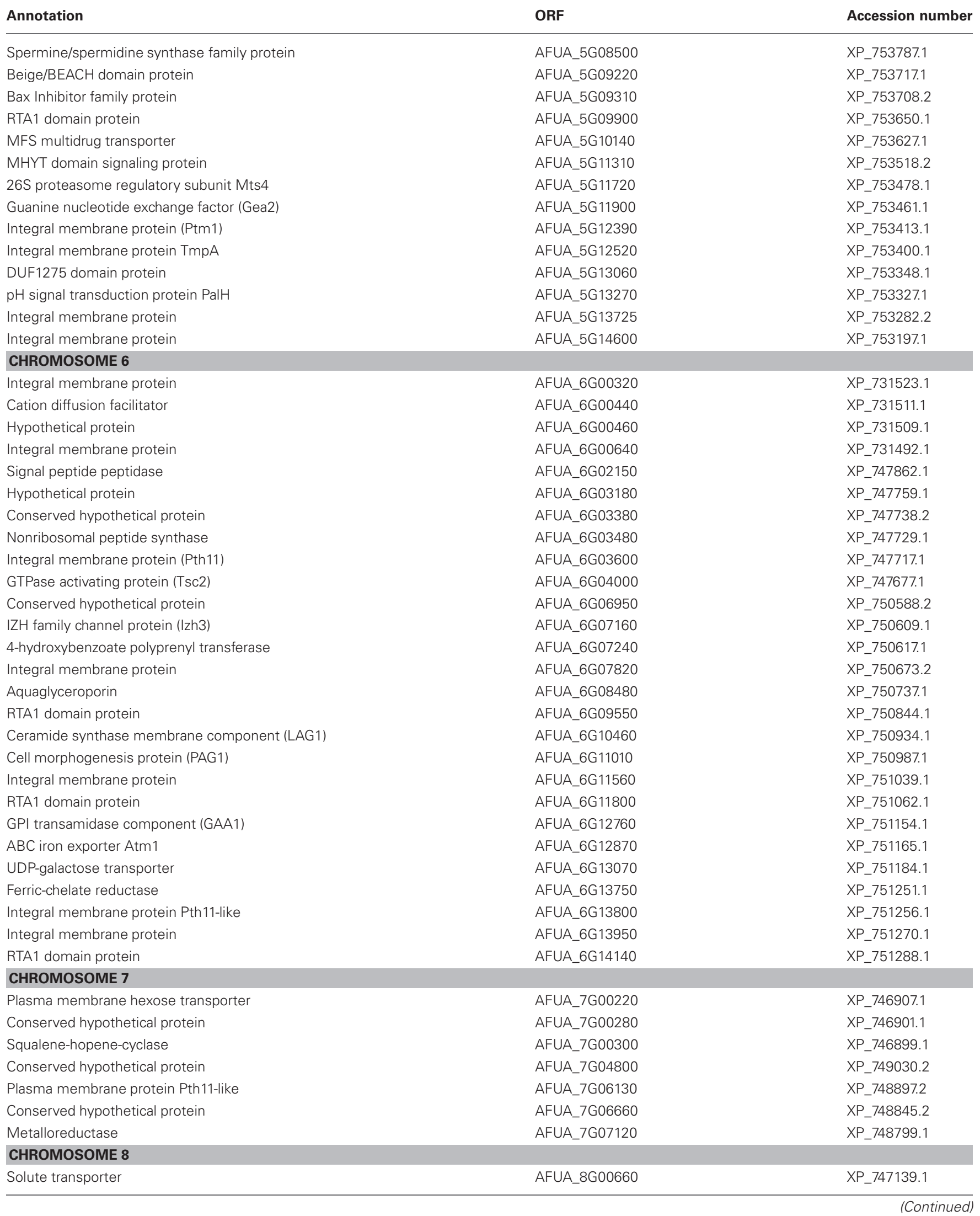




\title{
Table A1 | Continued
}

\begin{tabular}{lll}
\hline Annotation & ORF & Accession number \\
\hline Glycosyl transferase & AFUA_8G00680 & XP_747137.1 \\
Conserved hypothetical protein & AFUA_8G01300 & XP_747076.1 \\
GABA permease & AFUA_8G01450 & XP_747061.1 \\
NRPS-like enzyme & AFUA_8G01640 & XP_747042.1 \\
Conserved hypothetical protein & AFUA_8G01840 & XP_747022.2 \\
Conserved hypothetical protein & AFUA_8G02390 & XP_747208.2 \\
ZIP family zinc transporter & AFUA_8G04010 & XP_747263.1 \\
Integral membrane protein & AFUA_8G04560 & XP_747353.1 \\
Integral membrane protein & AFUA_8G05510 & XP_747364.1 \\
Chitin synthase F & AFUA_8G05630 & XP_747375.1 \\
RTA1 domain protein & AFUA_8G05740 & XP_747382.1 \\
Cellobiose dehydrogenase & AFUA_8G05805 & XP_747383.2 \\
DUF1295 domain protein & AFUA_8G05810 & XP_747399.1 \\
Toxin biosynthesis protein (Tri7) & AFUA_8G05970 & XP_747422.2 \\
Metalloreductase transmembrane component & AFUA_8G06210 & \\
\hline
\end{tabular}

Table A2 | Identity and annotations of $\boldsymbol{A}$. fumigatus histidine kinase receptors.

\begin{tabular}{|c|c|c|c|c|c|}
\hline Annotation & ORF & $\begin{array}{l}\text { Accession } \\
\text { number }\end{array}$ & $\begin{array}{l}\text { No. of } \\
\text { transmembrane } \\
\text { domains }\end{array}$ & Putative conserved domains & $\begin{array}{l}\text { Putative } \\
\text { group no. }\end{array}$ \\
\hline
\end{tabular}

CHROMOSOME 2

Sensor histidine kinase/ response regulator AFUA_2G00660 XP_001481640.1 4 $\mathrm{TcsB} / \mathrm{S} \ln 1$
(1) HiskA (Phospho-acceptor) domain
6
(2) Histidine kinase-, DNA gyrase B- and HSP90-like ATPase
(3) Histidine kinase-like ATPases
(4) cheY-homologous receiver domain

\begin{tabular}{|c|c|c|}
\hline $\begin{array}{l}\text { Two-component } \\
\text { osmosensing histidine } \\
\text { kinase (Bos1)-TcsC }\end{array}$ & AFUA_2G03560 & XP_749489.1 \\
\hline
\end{tabular}

osmosensing histidine

kinase (Bos1)-TcsC

\section{CHROMOSOME 3}

Sensor histidine kinase/

response regulator
AFUA $3 G 07130$

XP_754893.1

3

Sensor histidine kinase/ AFUA_3G12530 XP_754368.1 2

response regulator

\author{
AFUA_3G12530
}

$X P_{-}$754368.

2

3

(2)

Sensor histidine kinase/ AFUA_3G12550 XP_754366.1 9
response regulator

(1) PAS domain 5

(2) HiskA (Phospho-acceptor) domain

(3) Histidine kinase-like ATPases

(4) cheY-homologous receiver domain

5

$\begin{array}{ll} & \text { (3) GAF domain } \\ & \text { (4) HiskA (Phospho-acceptor) domain } \\ & \text { (5) Histidine kinase-like ATPases } \\ & \text { (6) cheY-homologous receiver domain }\end{array}$

\section{CHROMOSOME 4}

Sensor histidine kinase/

response regulator
AFUA_4G00320 XP_746424.2 2

(1) GAF domain

(2) HiskA (Phospho-acceptor) domain

(1) Multiple HAMP domains 3

(2) His kinase A (Phospho-acceptor) domain

(3) Histidine kinase-like ATPases

(4) cheY-homologous receiver domain

3
(1) HiskA (Phospho-acceptor) domain
(2) Histidine kinase-like ATPases
(3) cheY-homologous receiver domain
7

10
(1) Serine/threonine kinase domain
(2) AAA ATPase domain
(4) HiskA (Phospho-acceptor) domain
(6) cheY-homologous receiver domain 
Table A2 | Continued

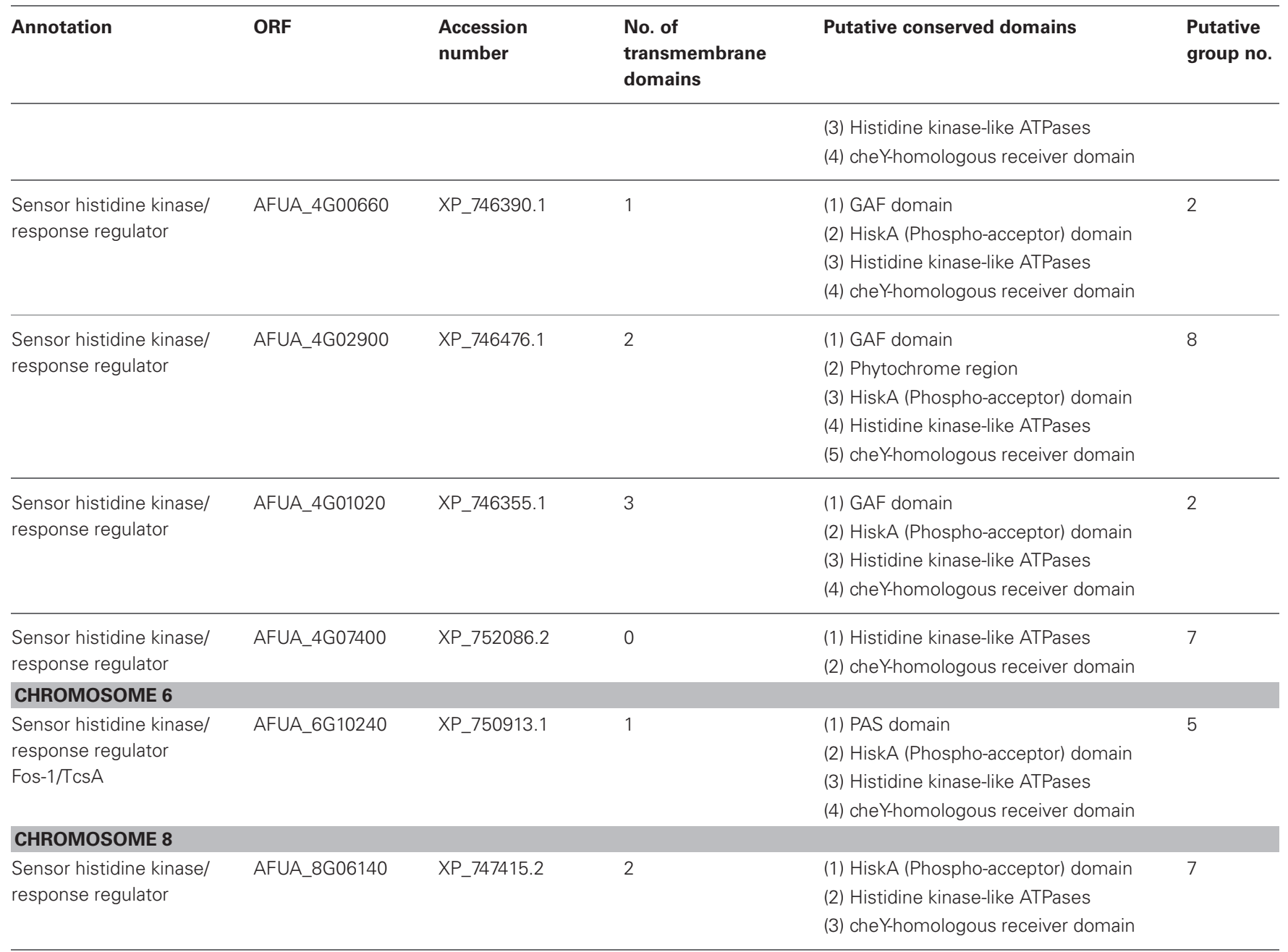

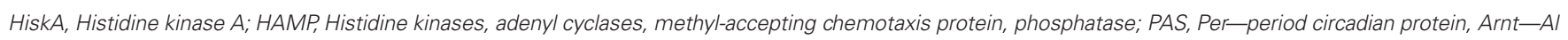

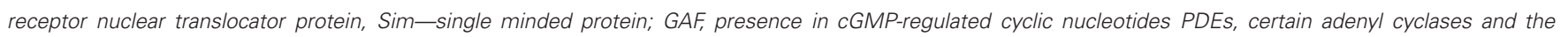
bacterial transcription factor FhIA; AAA, ATPases associated with diverse cellular activities. 